\title{
All-IP Based Ultra 3G Network/Service Development in a Competitive Market
}

\author{
Yutaka Yasuda \\ KDDI Corporation \\ 3-10-10, Iidabashi, Chiyoda-ku, Tokyo, Japan
}

\begin{abstract}
FMC (Fixed Mobile Convergence) is a key concept of NGN (Next generation Network) and KDDI announced the Ultra 3G concept based on allIP network configuration, 3 years ago. Now, the Ultra $3 \mathrm{G}$ concept has evolved toward FMBC (Fixed Mobile Broadcast Convergence). This talk introduces how KDDI has been swiftly and reliably developing all-IP based networks and FMBC services.
\end{abstract}

Keywords: All-IP Networks, FMBC, Modular Development.

\section{Introduction}

Recently, telecommunication carriers have considered that convergence of fixed and mobile communications, known as FMC (Fixed Mobile Communication) to be a key in creating new services and increasing the market. KDDI, a telecommunication carrier in Japan, has provided various kinds of FMC services such as "LISMO", a comprehensive music service that links "auc mobile phones with PCs (January 2006). In the future, KDDI will continue to meet customer demands by creating more convenient and attractive services and content along with the business strategy of "Fixed Mobile and Broadcast Convergence (FMBC)", targeting a fusion among fixed and mobile communications and broadcasting.

Within the competitive market in Japan, it is important to realize rapid and reliable developments of FMBC networks/services. After introducing the Ultra 3G concept, this invited talk describes how KDDI has been achieving rapid and reliable developments especially for FMBC services, before subsequently concluding with details of how KDDI provides reliability after developments.

\section{The Ultra 3G Concept}

Based on Ultra 3G, a next generation communication infrastructure concept, KDDI aims to build a fixed-mobile integrated network which provides integrated services, including third generation mobile phone systems such as "EV-DO Rev.A" (launched in December 2006), wireless LAN, new wireless systems such as mobile WiMAX and beyond 3G mobile network, and wired accesses such as ADSL and FTTH (Hikari-One). The concept is illustrated in Fig. 1. A service control system will be developed to provide seamless services with various forms of access means complementing 
each other. This system will be constructed in compliance with IP Multimedia Subsystem (IMS)/Multimedia Domain (MMD), which are standardized by 3GPP and 3GPP2, and expected to be globally deployed in the future.

This future infrastructure will enable customers to enjoy high-speed data services and high-quality multimedia services anytime and anywhere, within an optimum communication environment, without being aware of differences between fixed and mobile communications.

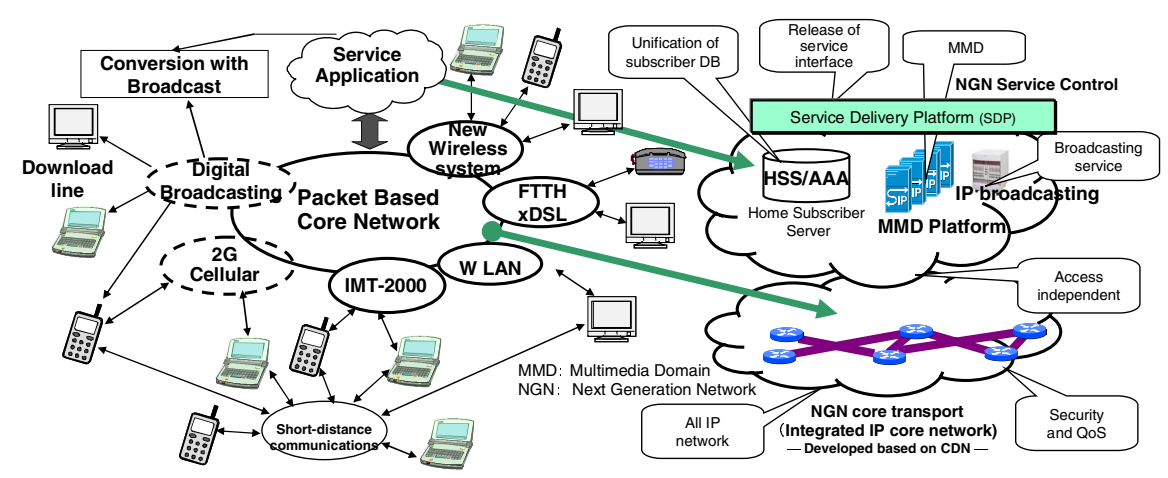

Fig. 1. Ultra $3 \mathrm{G}$ Concept

\section{Development Policies and Practices}

There are two important issues relating to network and service developments, respectively. The first concerns the reliability of all-IP networks compared to the circuit based legacy network. Legacy SS7 protocol suites are designed to achieve its reliability with coordination between protocol layers, while conversely, IP technology on which VoIP (Voice over IP) is based is relatively independent from both upper and lower layers. This is clearly one of the advantages of IP technology, but, at the same time, needs some additional knowledge and operational skills to achieve the same level of reliability. Also, VoIP networks are more centralized compared to the legacy equivalents, meaning that one problem may have a wider impact on the entire network. The KDDI VoIP system has improved through experiences over the years.

The second issue is rapid service development. The competitive market demands the rapidity of FMBC network/service developments without any loss of reliability. The left of this section shows some recent KDDI practices to introduce modular development to software systems comprising services rapidly and reliably delivered.

\subsection{Common Platform for Cellular Phone Software}

KDDI cellular phone consists of chipsets provided by QUALCOMM and other peripheral devices. In order to achieve portability of application programs on them, KDDI has been engaged in creating a common platform, KCP (KDDI Common Platform), as shown in the left part of Fig. 2. But due to device specific interfaces, vendor dependent implementations still existed and as the result application program developments were 
time consuming and were error prone. In order to achieve more portability, KDDI extended it to the more modular platform, $\mathrm{KCP}+$, as shown in the right part of Fig. 2. $\mathrm{KCP}+$ is developed on MSM7500 ${ }^{\mathrm{TM}}$ and $\mathrm{BREW}^{1}$ which are the chipset and its application programming interface respectively. It makes all kinds of programs common to any cellular phones. These include not only application programs such as browsers, mailers but also middleware software and OS (Operating System) itself.

Providing $\mathrm{KCP}+$ to vendors is expected to slash the development time and costs are expected to be reduced drastically. Besides vendors can also devote themselves to implementing their own functions related to UI (user interface) and/or design, etc. that differentiate their cellular phones to those of other vendors.

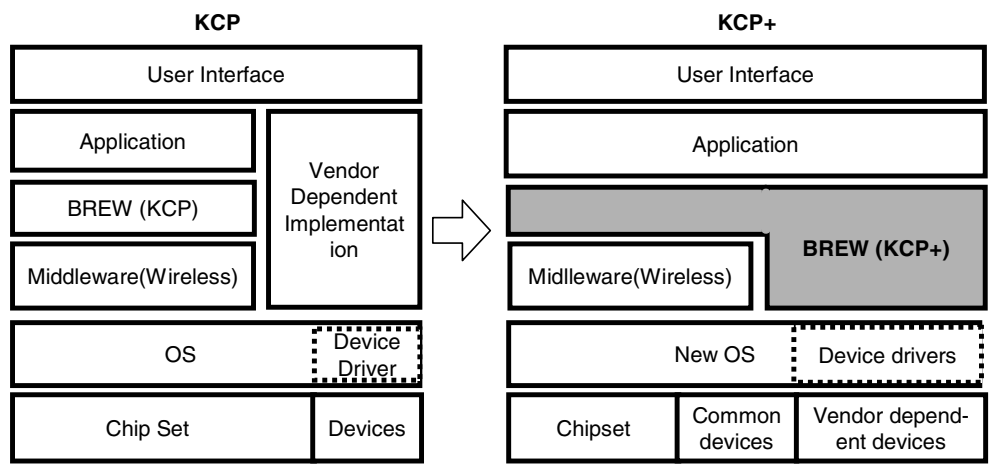

Fig. 2. Common Platform for Cellular Phone Software

\subsection{SOA Based Development}

BSSs (Business Support Systems) such as service, business and billing management systems used to be independently developed for individual services. Before the FMBC era, this style was not a problem; however, during the FMBC era, dependency among program modules of different BSSs has become problematic. Many new developments and updates which are performed due to rapidly changing business environments make them time consuming and error-prone, because a single change of an FMBC service has effects on several program modules of several BSSs. One possible solution is the introduction of an SOA (Service Oriented Architecture) based development.

As the initial step, KDDI has developed a BSS system that provides service management for Internet services over cellular phone networks. Before the system, known as ARIAL, was developed, service management systems were developed for individual services as shown in the left of Fig. 3. Conversely, functions common to all services such as "new order processes" and "call stop processes" are modeled as services of SOA-based development and are implemented in modular fashion using a BPM (Business Process Management) tool as shown in the right part of Fig.3. By introducing modular developments, a saving around $30 \%$ of the development time is possible while the throughput becomes 1.5 times as high as the previous BSS. Besides, failures that are localized to specific functions also improve reliability.

${ }^{1}$ BREW and MSM7500 TM are trademarks of QUALCOMM. 

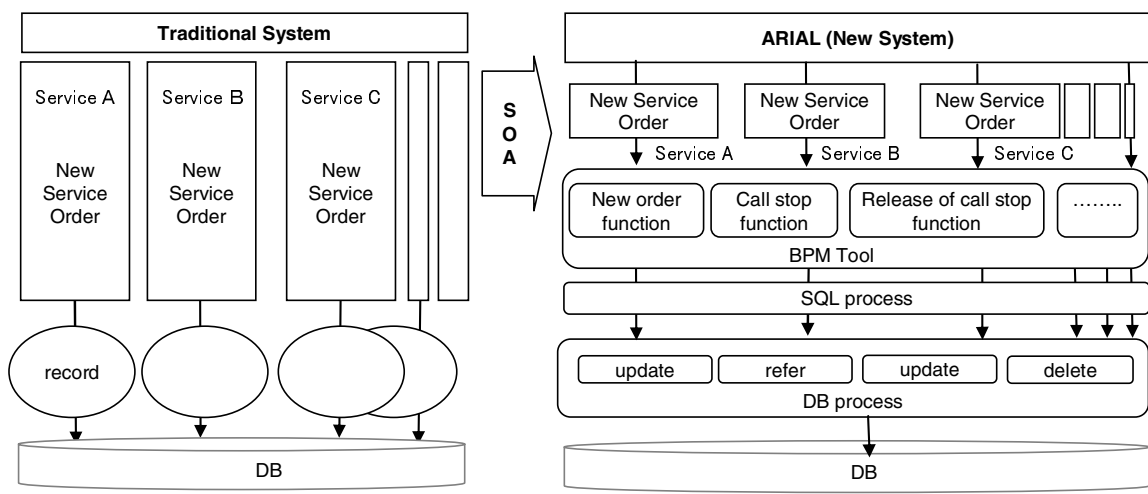

Fig. 3. SOA based Development

\section{Conclusion}

KDDI has been introducing FMBC core networks based on the Ultra $3 \mathrm{G}$ concept and FMBC services not only to survive the competitive environment, but also to enrich daily lives in Japan. This extended abstract shows the recent software development practices of KDDI. In addition KDDI is devoting itself to achieve flexible operations after developing FMBC network/service. The following are recent examples of techniques achieving flexible operations.

- Over-the-air software download techniques that distribute amendment of software bug and/or new software updates to cellular phones.

- Software-based device management techniques that collect and diagnose remote cellular phone logs.

- SLM (Service Level Management) techniques that measure the actively response times of servers and network equipments comprising services.

- Software radio techniques that enable programmability in wireless signal processing and reuse techniques that enable the software implementations of some wireless programmable devices to be ported to others at reasonable costs. 